Preprint typeset in JHEP style - HYPER VERSION

\title{
Tunneling of massive and charged particles from noncommutative Reissner-Nordström black hole
}

\author{
Kourosh Nozari* and Sara Islamzadeh ${ }^{\dagger}$ \\ Department of Physics, Faculty of Basic Sciences, University of Mazandaran, \\ P. O. Box 47416-95447, Babolsar, IRAN
}

\begin{abstract}
Massive charged and uncharged particles tunneling from commutative Reissner-Nordström black hole horizon has been studied with details in literature. Here, by adopting the coherent state picture of spacetime noncommutativity, we study tunneling of massive and charged particles from a noncommutative inspired Reissner-Nordström black hole horizon. We show that Hawking radiation in this case is not purely thermal and there are correlations between emitted modes. These correlations may provide a solution to the information loss problem. We also study thermodynamics of noncommutative horizon in this setup.
\end{abstract}

KEYwords: Black Hole, Noncommutative Geometry, Quantum Tunneling.

*knozari@umz.ac.ir

†s.islamzadeh@stu.umz.ac.ir 


\section{Contents}

1. Introduction

2. Motion of tunneling particles 2

3. Thermodynamics of noncommutative Reissner-Nordeström black hole

4. Conclusion 8

\section{Introduction}

After the discovery of Hawking radiation [1], a lot of attempts have been made to explore different aspects of this revolutionary achievement. There are some important questions in this regard: Is black hole radiation purely thermal? Are unitary and Lorentz invariance symmetries preserved at the quantum gravity level? What happens in the final stages of black hole evaporation? Are the information that were entered horizon at the time of star formation missing? Although, there is no perfect theory by now to answer these questions properly, various methods are presented to address such questions in recent years. One of these attempts is the strategy provided by Parikh and Wilczek [2]. In this approach, particle and antiparticle pairs are created and the particle tunnels through event horizon. Due to emission of this particle, total energy of black hole reduces. Conservation of energy requires that the event horizon radius reduces too. To deduce the black hole event horizon thermodynamics, Parikh and Wilczek utilized the WKB approximation. This approximation is actually justified since there is an infinite blue shift in the vicinity of the horizon. Indeed, the barrier through which tunneling occurs is induced by the emitted particle itself. They have considered the tunneling particle as a spherically symmetric shell that is ejected from black hole surface. This approach was the basis of a lot of research

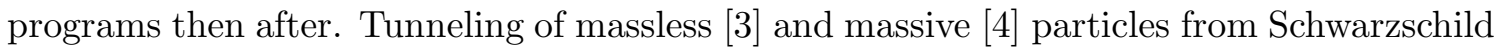
black hole and also noncommutative inspired Schwarzschild black hole [5] are studied. Recently, tunneling of massive and charged particles from Reissner-Nordström black hole horizon is reported too [6]. Also extensions to higher dimensional spacetime models are considered by some authors [7].

In recent years noncommutative quantum field theory has been attracted much attentions [8]. Noncommutativity is an intrinsic characteristic of manifold that implies the existence of a natural ultra-violet cutoff (or equivalently a minimal measurable length) in quantum field theory. Spacetime noncommutativity can be achieved naturally on certain backgrounds of string theory. In this viewpoint, description of the spacetime as a smooth commutative manifold becomes therefore a mathematical assumption no more justified by 
physics. It is then natural to relax this assumption and conceive a more general noncommutative spacetime, where uncertainty relations and spacetime discretion naturally arise. The noncommutativity of spacetime can be encoded in the commutator

$$
\left[\hat{x}^{i}, \hat{x}^{j}\right]=i \theta^{i j}
$$

where $\theta^{i j}$ is a real, antisymmetric and constant tensor, which determines the fundamental cell discretion of spacetime much in the same way as the Planck constant $\hbar$ discretizes the phase space. This was motivated by the need to control the divergences showing up in theories such as quantum electrodynamics. It has been shown that noncommutativity eliminates point-like structures in the favor of smeared objects in flat spacetime. As the authors have shown in Ref. [9], the effect of smearing is mathematically implemented as a substitution rule: position Dirac-delta function is replaced everywhere with a Gaussian distribution of minimal width $\sqrt{\theta}$. In this framework, they have chosen the mass density of a static, spherically symmetric, smeared, particle-like gravitational source as follows

$$
\rho_{\theta}(r)=\frac{M}{(2 \pi \theta)^{\frac{3}{2}}} \exp \left(-\frac{r^{2}}{4 \theta}\right)
$$

As they have indicated, the particle mass $M$ (or particle charge $Q$ ), instead of being perfectly localized at a point, is diffused throughout a region of linear size $\sqrt{\theta}$. This is due to the intrinsic uncertainty as has been shown in the coordinate commutators (1.1). This view point on spacetime noncommutativity has stimulated a lot of research programs in recent years, some of which can be seen in Refs [5, 0, 10]. In this paper we adopt this viewpoint to study tunneling of massive and charged particles from a noncommutative inspired Reissner-Nordström black hole horizon. We show that Hawking radiation in this case is not purely thermal and there are correlations between emitted modes that may provide a trace to address the information loss paradox. We study also thermodynamics of noncommutative horizon in this setup. Finally we investigate possible relation between charge and spacetime noncommutativity in this setup.

\section{Motion of tunneling particles}

In this section we consider Einstein field equations in coherent state noncommutative picture and we apply Peinlevé transformation to derive equations of motion of massive and charged particle in this setup. In coherent state picture of spacetime noncommutativity, a particle of mass $M$ and charge $Q$, instead of being perfectly localized at a point, is diffused throughout a region of linear size $\sqrt{\theta}$. In fact, the Dirac-delta function for pointlike structures is replaced everywhere with a Gaussian distribution of minimal width $\sqrt{\theta}$. In this framework, the mass and charge densities of a static, spherically symmetric, smeared, particle-like gravitational source can be modeled as follows

$$
\rho_{\text {mat }}(r)=\frac{M}{(4 \pi \theta)^{3 / 2}} e^{-\left(\frac{r^{2}}{4 \theta}\right)}
$$




$$
\rho_{e l}(r)=\frac{Q}{(4 \pi \theta)^{3 / 2}} e^{-\left(\frac{r^{2}}{4 \theta}\right)}
$$

In coordinate coherent state approach, noncommutative effects enters to energy-momentum tensor and current densities. Therefore, the Einstein-Maxwell field equations are preserved in usual form

$$
\begin{gathered}
R_{\nu}^{\mu}-\frac{1}{2} \delta^{\mu}{ }_{\nu} R=8 \pi\left(\left.T_{\nu}^{\mu}\right|_{m a t}+\left.T_{\nu}^{\mu}\right|_{e l}\right) \\
\frac{1}{\sqrt{-g}} \partial_{\mu}\left(\sqrt{-g} F^{\mu \nu}\right)=J^{\nu}
\end{gathered}
$$

Solving Einstein equations (2.1), the line element for a spherically symmetric system and the vector potential are as follows

$$
\begin{gathered}
d s^{2}=-g_{00} d t^{2}+g_{00}^{-1} d r^{2}+r^{2} d \Omega^{2} \\
A_{\mu}=h(r) \delta_{\mu}^{0}
\end{gathered}
$$

with

$$
g_{00}=1-\frac{2 M_{\theta}}{r}+\frac{Q_{\theta}^{2}}{r^{2}}
$$

and

$$
h(r)=-\frac{Q_{\theta}}{r}
$$

where by definition

$$
\begin{gathered}
M_{\theta}(r)=\frac{2 M}{\sqrt{\pi}} \gamma\left(\frac{3}{2}, \frac{r^{2}}{4 \theta}\right) \\
Q_{\theta}(r)=\frac{Q}{\sqrt{\pi}} \sqrt{\gamma^{2}\left(\frac{1}{2}, \frac{r^{2}}{4 \theta}\right)-\frac{r}{\sqrt{2 \theta}} \gamma\left(\frac{1}{2}, \frac{r^{2}}{2 \theta}\right)} .
\end{gathered}
$$

$\gamma$ in the above equations is incomplete lower Gamma function. The line element (2.3) has singularity at the horizons. To describe the tunneling process, we require a metric that is not singular on the horizon. The Painlevé coordinates transformation is a suitable tool to overcome this difficulty. With the Painlevé transformation [11] the metric (2.3) and vector potential (2.4) take the following forms respectively

$$
\begin{gathered}
d s^{2}=-g_{00} d t_{p}^{2}+d r^{2}+2 \sqrt{1-g_{00}} d t_{p} d r+r^{2} d \Omega^{2} \\
A_{\mu}=h(r) \delta_{\mu}^{0}-h(r) \frac{\sqrt{1-g_{00}}}{g_{00}} \delta_{\mu}^{1}
\end{gathered}
$$

With this transformation, horizon's singularity is removed and we can analyze tunneling process of particles through horizon. The Lagrangian equation of motion of a particle with mass $m$ and charge $q$ is obtained as follows

$$
\mathcal{L}=\frac{m}{2}\left(-g_{00} \dot{t}_{p}^{2}+\dot{r}^{2}+2 \sqrt{1-g_{00}} \dot{t}_{p} \dot{r}\right)+q h(r) \dot{t}_{p}-\frac{q h(r) \sqrt{1-g_{00}}}{g_{00}} \dot{r}
$$

where a dot indicates the derivative with respect to proper time $\tau$. By using the Euler-Lagrange equation $\left(\frac{\partial \mathcal{L}}{\partial q}-\frac{d}{d t} \frac{\partial \mathcal{L}}{\partial \dot{q}}=0\right)$ we find

$$
m g_{00} \dot{t}_{p}-m \sqrt{1-g_{00}} \dot{r}-q h(r) \equiv \mathcal{E}=\text { constant }
$$


To achieve the radial equation of motion of in the Painlevé coordinates, we need timelike trajectories that are given by

$$
g_{00} \dot{t}_{p}^{2}+\dot{r}^{2}+2 \sqrt{1-g_{00}} \dot{t}_{p} \dot{r}=-1
$$

Equations (2.8) and (2.9) can be solved simultaneously to obtain $\dot{r}$ and $\dot{t}$. Then equation of motion of a massive and charged particle in Peinlevé coordinates is obtained as follows

$$
\frac{d r}{d t_{p}}= \pm g_{00} \frac{\sqrt{(q h(r)+\mathcal{E})^{2}-m^{2} g_{00}}}{q h(r)+\mathcal{E} \pm \sqrt{1-g_{00}} \sqrt{(q h(r)+\mathcal{E})^{2}-m^{2} g_{00}}}
$$

This equation is the basis of our forthcoming analysis.

\section{Thermodynamics of noncommutative Reissner-Nordeström black hole}

In this section we study tunneling of massive and charged particles through horizon of a Reissner-Nordström black hole in a noncommutative space. Reissner-Nordström black hole can radiate through two ways

1- By radiating neutral particles. It causes the black hole to reach a Schwarzschild black hole in the final stage of evaporation.

2- By radiating charged particles. This happens through creation of charged particle and antipartile pairs.

We note that the second process leads to a black hole extreme configuration that is called the Reissner Nordström black hole remnant. As has been indicated in Ref. [12], electric field at the horizon in noncommutative picture is larger in value than the critical electric field and so black hole is able to create pair-charged particles. In which follows we focus on the later picture, that is, we consider radiation of pair-charged particles. As has been mentioned above, there are infinity blue shift in the vicinity of black hole event horizon. So, we can use the WKB approximation and calculate coefficients of transmission for a massive and charged particle that tunnels from inner radius to the outer radius. First, we consider imaginary part of the action for a particle that is coming from an initial state with $r_{\text {in }}$ to a final state with $r_{\text {out }}$

$$
\operatorname{Im} S \equiv \operatorname{Im} \int E d t=\operatorname{Im} \int_{r_{\text {in }}}^{r_{\text {out }}} p_{r} d r=\operatorname{Im} \int_{r_{\text {in }}}^{r_{\text {out }}} \int_{0}^{p_{r}} d p_{r} d r
$$

Using the Hamilton equation of motion, $d p_{r}=\frac{d H}{\dot{r}}$, we find

$$
\operatorname{Im} S=\operatorname{Im} \int_{r_{\text {in }}}^{r_{\text {out }}} \int_{m}^{\mathcal{E}} \frac{d H}{\dot{r}} d r=-\operatorname{Im} \int_{m}^{\mathcal{E}} \int_{r_{\text {in }}}^{r_{\text {out }}} \frac{d r}{\dot{r}} d \tilde{\mathcal{E}}
$$

Since the emitted particles are assumed to be massive, the lower limit of integral now is $m$ instead of being zero as for massless particles. Indeed, before tunneling, spherical shell of particle has an energy $\mathcal{E}$ and after crossing the event horizon, changed its energy to $\mathcal{E}-m$ and we'll represent effect of $m$ on the tunneling rate. In previous section we probed motion of a massive and charged particle in Painlevé's coordinates and we achieved 
equation of motion as given by Eq. (2.10). By expanding the metric around the event horizon, $g_{00}(r)=g_{00}\left(r_{\theta+}\right)+g_{00}^{\prime}\left(r_{\theta+}\right)\left(r-r_{\theta+}\right)+\ldots$, Eq. (2.10) takes the following form $\dot{r}= \pm g^{\prime}\left(r_{\theta+}\right)\left(r-r_{\theta+}\right) \frac{\sqrt{(q h(r)+\mathcal{E})^{2}-m^{2} g^{\prime}\left(r_{\theta+}\right)\left(r-r_{\theta+}\right)}}{(q h(r)+\mathcal{E}) \pm \sqrt{1-g^{\prime}\left(r_{\theta+}\right)\left(r-r_{\theta+}\right)} \sqrt{(q h(r)+\mathcal{E})^{2}-m^{2} g^{\prime}\left(r_{\theta+}\right)\left(r-r_{\theta+}\right)}}$

By substituting (3.2) in Eq. (3.1), integral has a pole at $r=r_{\theta}$. We solve this integral by using the calculus of residues. We find

$$
\operatorname{Im} S=\pi \int_{m}^{\mathcal{E}} \frac{2}{g_{00}^{\prime}\left(r_{\theta+}\right)} d \tilde{\mathcal{E}}
$$

By considering $g_{00}=\frac{\left(r-r_{\theta+}\right)\left(r-r_{\theta-}\right)}{r^{2}}$, the imaginary part of the action takes the following form

$$
\operatorname{Im} S=\pi \int_{m}^{\mathcal{E}} \frac{2 r_{\theta+}}{r_{\theta+}-r_{\theta-}} d \tilde{\mathcal{E}}
$$

To solve this integral we need to find the inner and outer Reissner-Nordstrom's event horizons in noncommutative geometry. So we $g_{00}\left(r_{\theta+}\right)=0$ to find

$$
r_{\theta \pm}\left(r_{\theta \pm}\right)=M_{\theta}\left(r_{\theta \pm}\right) \pm \sqrt{M_{\theta}^{2}\left(r_{\theta} \pm\right)-Q_{\theta}^{2}\left(r_{\theta} \pm\right)} .
$$

This equation has no analytical solution for $r_{\theta \pm}$. We can replace $r_{\theta \pm}$ by $r_{ \pm}$in lower incomplete Gamma function [13] to obtain the approximate event horizon radius as

$$
r_{\theta \pm}\left(r_{\theta \pm}\right) \cong r_{\theta \pm}(M, Q)=\mathcal{M}_{\theta \pm}(M, Q) \pm \sqrt{\mathcal{M}_{\theta \pm}^{2}(M, Q)-\mathcal{Q}_{\theta \pm}^{2}(M, Q)}
$$

So we find

$$
\begin{gathered}
\mathcal{M}_{\theta \pm}(M, Q)=M\left[\operatorname{erf}\left(\frac{M \pm \sqrt{M^{2}-Q^{2}}}{2 \sqrt{\theta}}\right)-\frac{M \pm \sqrt{M^{2}-Q^{2}}}{\sqrt{\pi \theta}} \exp \left(-\frac{\left(M \pm \sqrt{M^{2}-Q^{2}}\right)^{2}}{4 \theta}\right)\right] \\
\mathcal{Q}_{\theta \pm}(M, Q)=Q \sqrt{\operatorname{erf}^{2}\left(\frac{M \pm \sqrt{M^{2}-Q^{2}}}{2 \sqrt{\theta}}\right)-\frac{M \pm \sqrt{M^{2}-Q^{2}}}{\sqrt{2 \pi \theta}} \operatorname{erf}\left(\frac{M \pm \sqrt{M^{2}-Q^{2}}}{\sqrt{2 \theta}}\right)}
\end{gathered}
$$

where $\operatorname{erf}(x)$ is the Error Function. So, $r_{\theta \pm}$ in integral (3.3) becomes

$$
r_{\theta \pm}=\mathcal{M}_{\theta \pm}(M-\tilde{\mathcal{E}}, Q-q) \pm \sqrt{\mathcal{M}_{\theta \pm}^{2}(M-\tilde{\mathcal{E}}, Q-q)-\mathcal{Q}_{\theta+}^{2}(M-\tilde{\mathcal{E}}, Q-q)}
$$

We note also that, $r_{\text {in }}$ and $r_{\text {out }}$ are obtained as follows

$$
\begin{gathered}
r_{\text {in }}=\mathcal{M}_{\theta+}(M, Q)+\sqrt{\mathcal{M}_{\theta+}^{2}(M, Q)-\mathcal{Q}_{\theta+}^{2}(M, Q)} \\
r_{\text {out }}=\mathcal{M}_{\theta+}(M-\mathcal{E}, Q-q)+\sqrt{\mathcal{M}_{\theta+}^{2}(M-\mathcal{E}, Q-q)-\mathcal{Q}_{\theta+}^{2}(M-\mathcal{E}, Q-q)}
\end{gathered}
$$

and the integral pole lies between these to extremes. Substituting (3.5) into (3.6), the final form of the integral that should be calculated is as follows

$$
\operatorname{Im} S=\int_{m}^{\mathcal{E}} \frac{2 \mathcal{M}_{\theta+}^{2}(M-\tilde{\mathcal{E}}, Q-q)-\mathcal{Q}_{\theta+}^{2}(M-\tilde{\mathcal{E}}, Q-q)}{\sqrt{\mathcal{M}_{\theta+}^{2}(M-\tilde{\mathcal{E}}, Q-q)-\mathcal{Q}_{\theta+}^{2}(M-\tilde{\mathcal{E}}, Q-q)}} d \tilde{\mathcal{E}}+2 \int_{m}^{\mathcal{E}} \mathcal{M}_{\theta+}^{2}(M-\tilde{\mathcal{E}}, Q-q) d \tilde{\mathcal{E}}
$$


Analytical and exact solutions of the above integral is impossible. Thus, while we try to preserve noncommutativity effects, we expand the Gamma function around $\tilde{\mathcal{E}}$ to find some analytical results. In this situation, $\mathcal{M}_{\theta}$ and $\mathcal{Q}_{\theta}$ take the following forms respectively

$$
\mathcal{M}_{\theta+}(M-\tilde{\mathcal{E}}, Q-q) \simeq(M-\tilde{\mathcal{E}}) \eta
$$

and

$$
\mathcal{Q}_{\theta+}(M-\tilde{\mathcal{E}}, Q-q) \simeq(Q-q) \zeta
$$

where by definition,

$$
\eta=\frac{2}{\sqrt{\pi}} \gamma\left(\frac{3}{2}, \frac{\left(M+\sqrt{M^{2}-Q^{2}}\right)^{2}}{4 \theta}\right)
$$

and

$$
\zeta=\frac{1}{\sqrt{\pi}} \sqrt{\gamma^{2}\left(\frac{1}{2}, \frac{\left(M+\sqrt{M^{2}-Q^{2}}\right)^{2}}{4 \theta}\right)-\frac{M+\sqrt{M^{2}-Q^{2}}}{\sqrt{2 \theta}} \gamma\left(\frac{1}{2}, \frac{\left(M+\sqrt{M^{2}-Q^{2}}\right)^{2}}{2 \theta}\right)}
$$

Eventually, substituting Eqs. (3.7) and (3.8) into Eq. (3.6) we obtain the imaginary part of the action as follows

$$
\begin{aligned}
\operatorname{Im} S & =\pi\left[\eta(M-m)^{2}-\eta(M-\mathcal{E})^{2}\right. \\
& +(M-m) \sqrt{\eta^{2}(M-m)^{2}-\zeta^{2}(Q-q)^{2}} \\
& \left.-(M-\mathcal{E}) \sqrt{\eta^{2}(M-\mathcal{E})^{2}-\zeta^{2}(Q-q)^{2}}\right]
\end{aligned}
$$

Since $\Gamma=e^{-\frac{\mathcal{E}}{T}} \sim e^{-2 \operatorname{Im} S}$, existence of nonlinear terms in the imaginary part of the action requires correlation between the emitted modes. In other words, now two different particles with energies $\mathcal{E}_{1}$ and $\mathcal{E}_{2}$ are correlated. This means that $\Gamma_{\mathcal{E}_{1}+\mathcal{E}_{2}} \neq \Gamma_{\mathcal{E}_{1}}+\Gamma_{\mathcal{E}_{2}}$. Therefore, emission rate is no longer purely thermal and part of information can be supplied in correlations between emitted modes. Moreover, transition coefficient depends on energy, mass and charge of emitted particle and also spacetime noncommutativity parameter.

To obtain temperature, we expand Eq. (3.11) with respect to $m, \mathcal{E}$ and $q$ to find

$$
\begin{aligned}
\operatorname{Im} S & =\pi \frac{\left(\eta M+\sqrt{\eta^{2} M^{2}-\zeta^{2} Q^{2}}\right)^{2}}{\sqrt{\eta^{2} M^{2}-\zeta^{2} Q^{2}}}\left\{\left(1-\frac{q \zeta^{2} Q\left(3 \eta^{2} M^{2}-\zeta^{2} Q^{2}\right)}{\left(\eta^{2} M^{2}-\zeta^{2} Q^{2}\right)\left(\eta M+\sqrt{\eta^{2} M^{2}-\zeta^{2} Q^{2}}\right)^{2}}\right)(\mathcal{E}-m)\right. \\
& \left.+\frac{2 \eta\left(\eta^{2} M^{2}-\zeta^{2} Q^{2}\right)^{\frac{3}{2}}+2 \eta^{4} M^{3}-3 \eta^{2} \zeta^{2} M Q^{2}}{\left(\eta^{2} M^{2}-\zeta^{2} Q^{2}\right)\left(\eta M+\sqrt{\eta^{2} M^{2}-\zeta^{2} Q^{2}}\right)}\left(m^{2}-\mathcal{E}^{2}\right)+\ldots\right\}
\end{aligned}
$$

We see that when the emitted particle is massive, the mass of the particle appears explicitly in tunneling rate. Since spacetime at infinity tends to the Minkowski spacetime, the observer at infinity detects particles on-shell. Also, since $\mathcal{E}>m$, the condition $\operatorname{Im} S>0$ is satisfied. Therefore, the emitted particle mass causes a shift in tunneling rate through noncommutative horizon. Now the modified Hawking temperature for Reissner-Nordström black hole in noncommutative space is as follows

$$
T=\frac{\sqrt{\eta^{2} M^{2}-\zeta^{2} Q^{2}}}{2 \pi\left(\left(\eta M+\sqrt{\eta^{2} M^{2}-\zeta^{2} Q^{2}}\right)^{2}-\zeta^{2} q Q \frac{3 \eta^{2} M^{2}-\zeta^{2} Q^{2}}{\eta^{2} M^{2}-\zeta^{2} Q^{2}}\right)}
$$


The effect of noncommutativity is hidden in $\eta$ and $\zeta$ that are defined by Eqs. (3.9) and (3.10) respectively. Note that we considered the general case of tunneling of massive and charged particles though event horizon of a noncommutative Reissner-Nordström black hole. If we set $\theta=0$, temperature reduces to the commutative case result that has been reported in Ref. [6] and if we set $q=0$, we receive the classical commutative space Hawking temperature $T_{H}=\frac{\sqrt{M^{2}-Q^{2}}}{2 \pi\left(M+\sqrt{M^{2}-Q^{2}}\right)^{2}}$. Also, our result is consistent with noncommutative case with $Q=0$ that is reported in Ref. [5]. Indeed, our result contains all limiting cases properly.

Figure 1 shows the Hawking temperature of a Reissner-Nordström black hole versus its mass in noncommutative space and for different black hole charge. As we see, the final state temperature decreases as the black hole charge increases. We note that black hole evaporates through radiation of charged particle-antiparticle pairs until it reaches a remnant with maximal temperature. In Ref. [12] analytical results are obtained for minimum mass of Reissner-Nordström black hole in noncommutative geometry. This minimal mass is due to considering of Gaussian distribution in noncommutative geometry for mass and charge. As figure 1 illustrates, temperature of Reissner-Nordström black hole remnant is less than the temperature of Schwarzschild black hole remnant. So, the Reissner-Nordström black hole remnant is colder than the Schwarzschild black hole remnant. As another important outcome, the noncommutativity effect becomes more effective for small charges. We note that although our results agree with results obtained in Ref. [12], but we obtained these results in a different manner through tunneling method.

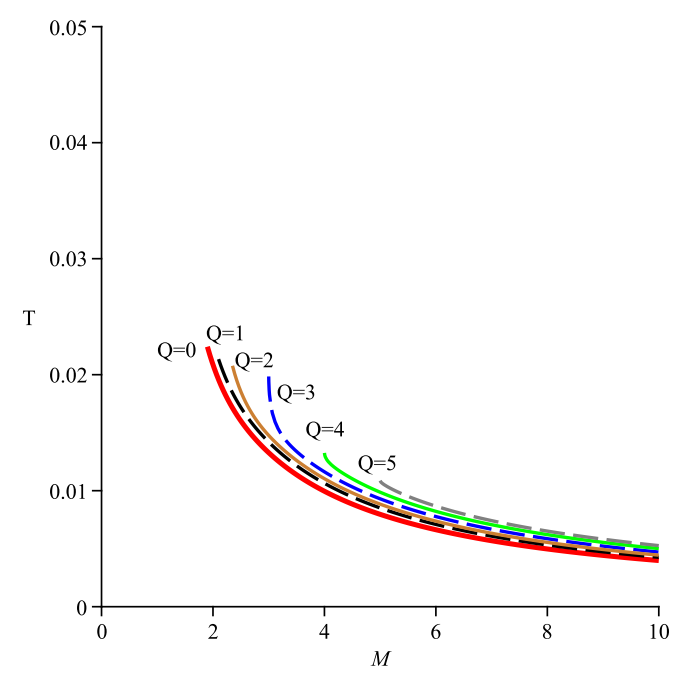

Figure 1: Temperature of a noncommutative Reissner-Nordström black hole versus its mass for different charge.

To compare with commutative case, we present figure 2 that is obtained for a commutative Reissner-Nordström black hole. The authors in Ref. 14] have shown that a non- 
commutative Schwarzschild black hole has features very similar to a commutative ReissnerNordström black hole. They have concluded that there is a close relation between charge and noncommutativity. Here we see that Reissner-Nordström black hole in commutative space has a remnant in the final stage of its evaporation just due to its charge. In other words, charge by itself prevents total evaporation of a Reissner-Nordström black hole and this is much similar to say that noncommutativity prevents a Schwarzschild black hole from total evaporation. This shows that existence of black hole charge can address at least part of the temperature divergence at final stage of evaporation and information loss problems by prediction of a final state non-zero mass remnant. We note also that the remnant mass for commutative and noncommutative Reissner-Nordström black hole are different. This is the case also for temperature.

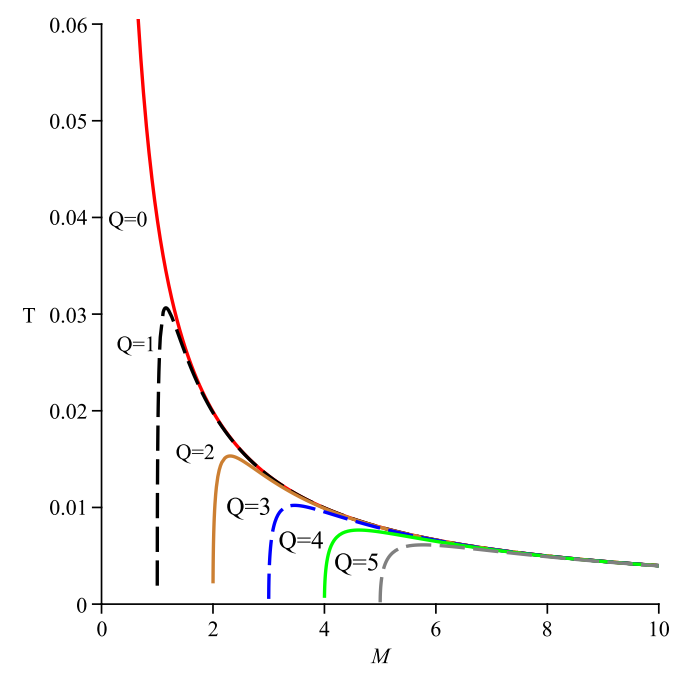

Figure 2: Temperature of a commutative Reissner-Nordström black hole versus its mass for different charges.

\section{Conclusion}

Within the coherent state picture of spacetime noncommutativity, we have studied tunneling of massive, charged particles in noncommutative horizon of a Reissner-Nordström black hole. We applied the standard Parikh-Wilczek tunneling method by adopting the Peinlevé transformation in order to remove singularities of metric. We have shown that tunneling rate is dependent on the energy, mass and charge of tunneling particles and also the noncommutativity parameter $\theta$. We have shown that noncommutative Reissner-Nordström black hole radiates through Hawking process until it reaches a remnant whose final temperature and mass depends on the noncommutativity parameter and total charge of the black hole. Also we have shown that the Reissner-Nordström black hole remnant is colder than the Schwarzschild black hole remnant and the noncommutativity effect becomes more effective for small black hole charges. Finally by comparing evaporation process of a commutative Reissner-Nordström black hole with a noncommutative Schwarzschild black hole 
we concluded that black hole charge by itself can prevent total evaporation of ReissnerNordström black hole much in the same way that spacetime noncommutativity prevents total evaporation of a Schwarzschild black hole. This feature is in agreement with previous finding in Ref. 14] that there is some nontrivial connection between charge and noncommutativity.

\section{References}

[1] S. W. Hawking, Comm. Math. Phys. 43, 199 (1974).

[2] M. K. Parikh and F. Wilczek, Phys. Rev. Lett. 85, 5042 (2000).

[3] M. Arzano, A. J. M. Medved and E. C. Vagenas, JHEP 0509, 037 (2005)

K. Nozari and S. H. Mehdipour, Europhys. Lett. 84, 20008 (2008)

[4] J. Zhang and Z. Zhao, Nucl. Phys. B 725, 173 (2005).

[5] K. Nozari and S. H. Mehdipour, Class. Quantum Grav. 25, 175015 (2008) Y. -G. Miao, Z. Xue and S. -J. Zhang, Gen. Relativ. Gravit. 44, 555 (2012).

[6] Y. -G. Miao, Z. Xue, S. -J. Zhang, Europhys. Lett.96, 10008, (2011).

[7] T. G. Rizzo, JHEP 09, 021 (2006)

K. Nozari and S. H. Mehdipour, JHEP 03, 061 (2009)

K. Nozari and S. H. Mehdipour, Commun. Theor. Phys. (Beijing, China) 53, 503 (2010).

[8] S. H. Snyder, Phys. Rev. 71, 38 (1974)

N. Seiberg and E. Witten, JHEP 09, 032 (1999)

M. R. Douglas and N. A. Nekrasov, Rev. Mod. Phys. 73, 977 (2001)

R. J. Szabo, Phys. Rep. 378, 207 (2003)

M. Chaichian, et al., Eur. Phys. J. C 29, 413 (2003)

A. Micu and M. M. Sheikh-Jabbari, JHEP 0101, 025 (2001).

[9] A. Smailagic and E. Spallucci, J. Phys. A 37, 1 (2004)

A. Smailagic and E. Spallucci, J. Phys. A 37, 7169 (2004)

A. Smailagic and E. Spallucci, J. Phys. A 36, L467 (2003)

A. Smailagic and E. Spallucci, J. Phys. A 36, L517 (2003).

[10] P. Nicolini, A. Smailagic and E. Spallucci, ESA Spec. Publ. 637, 11 (2006)

P. Nicolini, J. Phys. A 38, L631 (2005)

P. Nicolini, A. Smailagic and E. Spallucci, Phys. Lett. B 632, 547 (2006)

S. Ansoldi, P. Nicolini, A. Smailagic, E. Spallucci, Phys. Lett. B 645, 261 (2007)

E. Spallucci, A. Smailagic and P. Nicolini, Phys. Lett. B 670, 449 (2009)

Y. S. Myung and M. Yoon, Eur. Phys. J. C 62, 405 (2009)

M. I. Park, Phys. Rev. D 80, 084026 (2009)

R. Garattini and F. S. N. Lobo, Phys. Lett. B 671, 146 (2009)

P. Nicolini and E. Spallucci, Class. Quant. Grav. 27, 015010 (2010)

I. Arraut, D. Batic and M. Nowakowski, Class. Quant. Grav. 26, 245006 (2009)

P. Nicolini, Int. J. Mod. Phys. A 24, 1229 (2009)

I. Arraut, D. Batic and M. Nowakowski, J. Math. Phys. 51, 022503 (2010)

D. Batic and P. Nicolini, Phys. Lett. B 692, 32 (2010)

A. Smailagic and E. Spallucci, Phys. Lett. B 688, 82 (2010)

W. -H. Huang, [arXiv:1003.1040]. 
[11] P.Painlevé, C. R. Acad. Sci. (Paris) 173677 (1921).

[12] S. Ansoldi, P. Nicolini, A. Smailagic, E. Spallucci, Phys. Lett. B 645, 261 (2007).

[13] S. H. Mehdipour, Int. J. Mod. Phys. A25, 5543 (2010)

S. H. Mehdipour, Phys. Rev. D 81, 124049 (2010)

S. H. Mehdipour, Can. J. Phys., 90, 425 (2012).

[14] K. Nozari, B. Fazlpour, Acta Phys. Polon. B39,1363 (2008). 